\title{
Research on the Cloud Platform Development of Visual Communication under the Internet+ Model
}

\author{
Guannan Chen \\ Academy of Arts, Jilin Engineering Normal University, Changchun, 130052, China
}

Keywords: cloud platform; professional work; visual communication; internet + model

\begin{abstract}
The advent of the Internet age brings about the transformation of design media, design methods and design forms. Compared with traditional visual communication design, the visual communication design under the Internet + model has unique advantages and characteristics. The paper analyses the system structure of cloud platform of professional work of visual communication and the key technologies under the Internet+ model, including cloud platform based on container technology, coding system based on generic server and recommendation engine based on big data analysis to provide some references for relevant researchers.
\end{abstract}

\section{Introduction}

Visual communication design, which uses visual symbols to convey information design, can be considered as the extension and expansion of graphic design and graphic design. Visual communication design takes mass media as the carrier, and there is a close relationship between them. The development of carrier will lead to the transformation of design style. The visual communication design has also experienced several stages, such as commercial art, arts and crafts, printing art design, decoration design, plane design, and so on, and finally became the visual medium as the carrier, using visual symbols to express and convey information. In the age of Internet, the carrier of visual communication has been revolutionized, and the mobile Internet based on the Internet, as a new mass media, has the characteristics of quick, timely, cheap, good interactivity, wide spread and anytime and anywhere, which provides good conditions for the transmission of visual communication design. Visual communication design based on mobile Internet transmission will be restricted by mobile Internet technology. With the development of mobile Internet, the terminal that is rooted in mobile devices will have a new form of multimedia display, and these new media display ways will affect the design of visual communication, making the visual communication design a new style and characteristics. Cloud computing has significant advantages in business innovation, service provision, resource optimization and so on. Cloud computing, based on large scale computing nodes, can not only improve the utilization of servers, provide high performance computing and mass data storage, but also integrate and utilize existing infrastructure to reduce the capital investment in the construction and maintenance of data centers. It is an important technique to solve a series of problems encountered in the construction of information technology. The means of operation. Therefore, the construction of the visual communication operation cloud platform for the fusion of three networks will certainly bring a significant cost saving and service quality improvement, reduce the cost and improve the user experience.

\section{System Structure of Cloud Platform of Visual Communication under the Internet+ Model}

Similar with the general cloud platform, the system structure of cloud platform of professional work of visual communication under the internet+ model also includes Iaas, Paas and Saas, which is shown in Figure 1. 


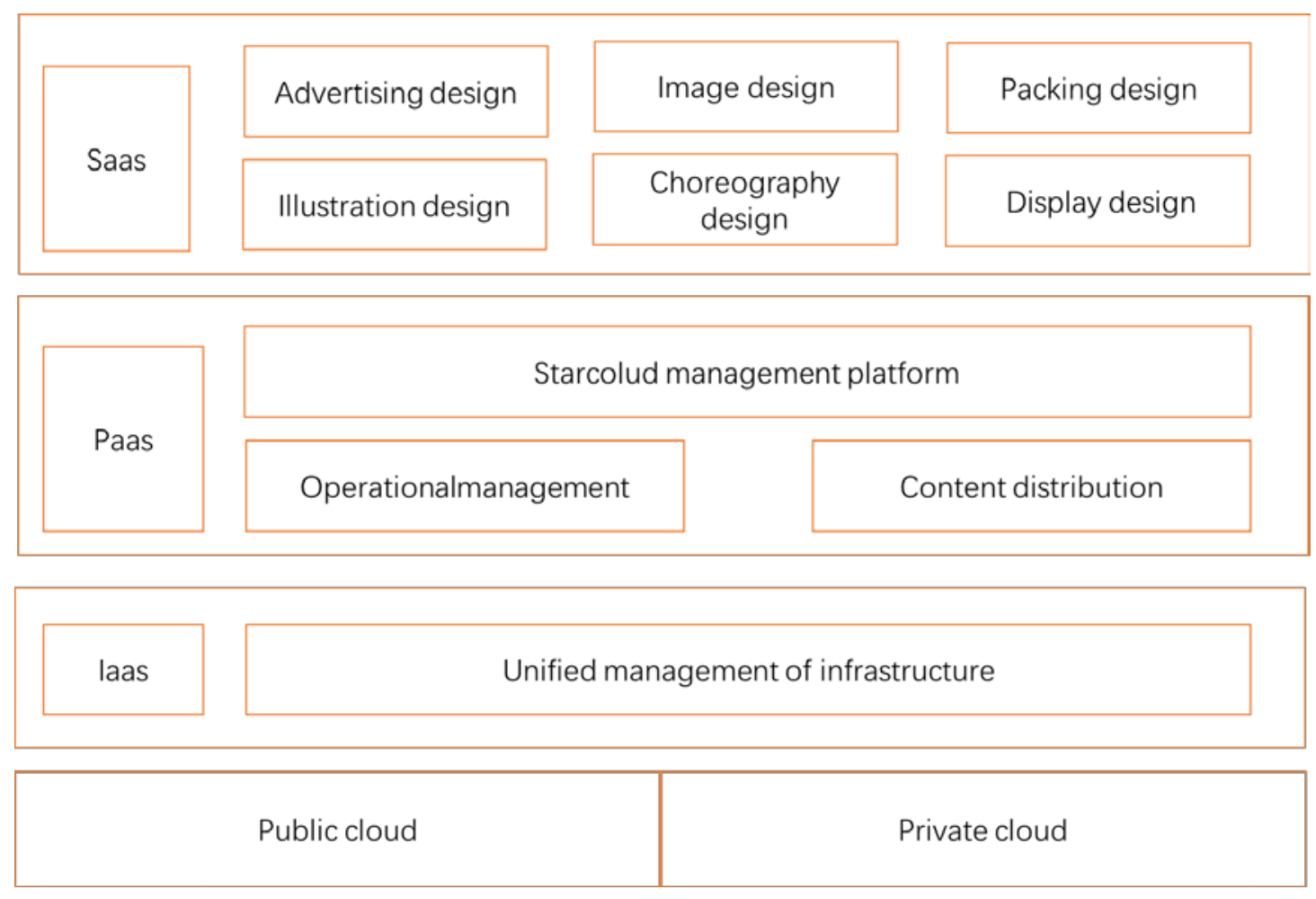

Figure 1. Iaas, Paas and Saas of the system structure of cloud platform

Iaas. The infrastructure platform uses virtualization technology to manage the traditional IT infrastructure, providing unified computing and data transmission capabilities for the upper business system. Through the virtualized computing subsystem and the virtualized network subsystem, the universal server and network equipment are pooled to form the network resource pool of CPU, GPU, memory and hard disk. Virtualization technology mainly includes virtualization in network, data storage, server and so on. After the server virtualization, the operating system is abstracted to be realized. The system itself is separated from the physical hardware and becomes a virtual machine. The system is migrated in real time, fast, and the service is more secure and efficient, and the utilization of the server is improved. Data storage virtualization is mainly to isolate the actual data storage between a number of relatively independent logical storage spaces, and then merge into the storage pools with high performance. The logical storage space after the virtualization is used in the form of software. In this case, both the operating system and the component application data storage are changed.

Paas. Cloud service is a kind of active service. Registration service is very important in many services offered by itself. It is a bridge between service providers and users. So on the visual communication cloud platform, cloud services can be seen as a service type, provided by the service provider in the connection of the registration service, the recipient is the user, and the visual communication cloud platform can support the release of service and the order of the service. As a service user, the user can view the service information issued by the service provider on the visual communication cloud platform, and choose the service to be purchased in line with its own service, and use the service on the Internet. Cloud service management integrates many visual communication software, which can serve as a service commodity on the cloud platform. Users can be both personal and visual communication institutions or schools. The interactivity, centrality and convenience of cloud service management make the use of the related visual communication resources more reasonable and cheaper. For all types of users, the cost of using software is also greatly reduced. The cloud platform management system carries on the unified scheduling management to the resource pool. According to the requirements of the upper service system, the resources of the resource pool are composed of the virtual cloud host, which provides the computing and data transmission for the hierarchical business system. The cloud management platform contains mirror image repository to store the image of each business system, and automatically deploy the business system at the same 
time to build the virtual cloud host, and realize the level expansion of the business system. In the process of cloud service management, the creation of database and virtual directory ensures the sharing and use of data information. Any user who buys the same software can share all the information stored in the database through the cloud platform. To create database, we need not only create new database, but also design new database table and initialize database.

Saas. Based on the visual communication service, and then according to the industry needs to access the industry applications, such as advertising design, image design, packaging design, illustration design, layout design, display design and so on through the unified business authentication system access, unified portal to achieve a unified display, to ensure the orderly and standardized access of multi service. Advertising design refers to the intermediate process from creativity to production. Advertising design is a combination of five elements, including advertising theme, creativity, language, image, and foil. The ultimate goal of advertising design is to achieve eye catching through advertisements. The image designer profession is the main application discipline in the world in recent years. Image processing and multimedia technology have entered thousands of households, and the corresponding software is emerging one after another. Packaging design covers a wide range of elements, including packaging design materials, modeling, printing, visual transmission and many other elements. Packaging design not only follows the rule of plane design, but also reflects commodity information, product image and so on. Illustration is the image that is expressed by the pattern. In line with the principle of unity of aesthetics and practicality, we try to make the lines clear and clear and convenient to make. Illustration is the language that the world can use. Its design is usually divided into characters, animals and commodity images in commercial applications. Arrangement design is a kind of organizational structural design based on the elements of visual information and media media elements. It is based on the characters, images, figures, symbols, colors, scales, space and other elements and specific information needs, organized, composing and typesetting according to the aesthetic principle and the character of human reading. Mask has a certain sense of visual beauty, suitable for reading habits, causing people's interest in reading. Exhibition design is a comprehensive art design; whose main body is merchandise. The exhibition design creates the exhibition space, which not only contains the intention to explain the exhibits, but also enables the audience to participate in it, so as to achieve the goal of perfect communication.

\section{Key Technologies of Cloud Platform of Visual Communication under the Internet+ Model}

\subsection{Cloud Platform Based on Container Technology.}

Based on Docker container technology, packaging applications, relationships and configuration information will greatly enhance the portability of applications and realize cross cloud management. At the same time, it can be used to implement like capabilities to provide more computing resources for users with more saving hardware resources: because of the use of operating system based virtualization technology, it uses namespace technology to distinguish different containers, and when running a container, the process, network interface, process inside the container, and process Inter communication resources, mount points and system kernel are all in the namespace of the container, which ensures isolation. The cloud management platform based on container technology will provide users with high performance container cluster management solutions: support flexible expansion, direct expansion, grayscale upgrading, service discovery, service arrangement, error recovery and performance monitoring, so that operators can quickly deploy services and easy operation and maintenance services. The key to visual design is to grasp the specific information and use visual creativity and inspiration. It is also the main driving force of visual impact, influence and infection. After entering the Internet era, the manifestation of visual communication, digitalization, multimedia and integration is the main expression of current media innovation. The popularity of computers has caused the designer's fierce competition for information applications. The amount of information, the speed of information transmission, the speed of information processing, and the efficiency of information application are increasing at a double rate. For the field of art and design, designers, 
through their inspiration and experience, will get information from the web by software processing and design into visual design works. Visual communication design has the visual stimulation, and gradually changes people's life habits, values and aesthetic views through information and digital media, and becomes an important source of people's knowledge.

\subsection{Coding System Based on Generic Server.}

The general server can quickly deploy in all kinds of common server cloud platforms, dynamically scheduling computing nodes in the cloud platform through adaptive computing capability prediction algorithm, and providing high quality coding transcoding services with minimal computing resources. The coding system should adopt multi-level task allocation and parallel framework, and speed up and optimize the coding process by using coding information, so as to dynamically allocate and use computing resources on the platform of high computing density to carry out real-time coding and transcoding tasks of video. The visual communication design in the Internet era shows strong communication characteristics. Strong communication means that the visual communication design is interactive through the electronic media and the audience, which transcends the traditional static characteristics of the media, which is embodied in the interactive advertising, the virtual product display and so on. The visual communication design in the Internet era changes the fixed role of the former information publisher and the recipient, and the receptor can have more independent options and influence the information publisher through feedback and other forms, so that the information can be displayed at any time. In the era of internet, information is more inclusive, through network and other technology, people can easily obtain any aspect of the required information, from real-time news to professional knowledge skills, can be obtained through high-speed media. At the same time, some information can be displayed in the form of images, providing better understanding and acceptance for the audience. Thanks to the media technology innovation, early restrictions such as paper print media have been overcome. In the era of Internet, information carrying capacity of visual communication design is more powerful by means of images, images and other media.

\subsection{Recommendation Engine Based on Big Data Analysis.}

Considering the users' commonalities and fully respecting different user's personalized choices, we can greatly improve user's viewing experience. By using machine learning, statistical algorithm and other technologies, a recommendation engine based on big data analysis can be realized, and valuable knowledge can be found from a large number of data. The recommendation engine will be based on the analysis of content data, user data and behavioral data, according to different business needs, to form different mechanisms of recommendation, including active recommendation, classification recommendation, fusion recommendation and so on. It will be applied to advertising release, video recommendation and so on. By improving the user experience, the conversion rate and the viscosity of the user can be improved. Improve content utilization and purchase rate, and ultimately improve operator's service quality, competitiveness and revenue. The application of Internet thinking in visual communication design brings people more intuitive and vivid information experience. The excessive pursuit of information efficiency in the Internet era makes the visual communication design pay attention to the chart of data, and make the design work lack of interesting and emotional factors. The visual communication design in the internet era has failed to form a new design thinking. The development of the new media has brought a more convenient environment but it has also failed to cause new design thinking.

\section{Conclusion}

In the era of internet, visual language elements exist in all visible objects. If we can study the impact of visual psychology to visual communication design and develop new directions for visual communication design through the design of these elements, we will be able to better use visual communication design in the context of contemporary society. This paper explores the development of cloud platform development of professional work of visual communication under the internet+ model. We can explore the cloud platform development based on the 3D virtual visions. 


\section{References}

[1] Huo Ju, Wang Xingshun, Wang Yongkun, et al. Development of Internship Platform for Visual Positioning [J]. Experiment Science and Technology, 2016, 14(3): 5-9.

[2] Zhang Yuan. Research on Sign System Design Based on 3D Virtual Vision [J]. Computer Simulation, 2016, 33(6): 382-385.

[3] Chen Rong, Liu Jie. Design and implementation of specific staff retrieval platform based on intelligent vision [J]. Modern Electronics Technique, 2017, 40(14): 102-105.

[4] Li Jingyu, Lu Tianyi. Research on the Design Method and Form of Visual Communication in Internet Era [J]. Design, 2016(17): 61-63. 